\title{
Sputum inflammatory cells in COPD patients classified according to GOLD 2011 guidelines
}

\section{To the Editor:}

Recently the definition of chronic obstructive pulmonary disease (COPD) severity, stated in 2001 by Global Initiative for Chronic Obstructive Lung Disease (GOLD) guidelines and based on forced expiratory volume in $1 \mathrm{~s}\left(\mathrm{FEV}_{1}\right)$ evaluation, has been considered inadequate to recognise all the multifaceted aspects of the disease. To overcome this limitation, 2011 GOLD recommendations suggested a new approach, which stratified COPD patients in four groups of severity (A, B, C, D) according to the level of symptoms (as assessed by either the modified Medical Research Council (mMRC) score or the COPD Assessment Test), the degree of airflow limitation (expressed as percentage of predicted forced expiratory volume in $1 \mathrm{~s}$ (FEV1) value) and the number of exacerbations in the previous year [1]. It is still matter of debate if this new classification helps to better understand the disease and improve COPD treatment and prognosis. Studies on the existing database have been published comparing the old with the new GOLD classifications, showing that GOLD 2011 classification adds new details on the phenotyping and on the prognosis of COPD [2-5]. Despite the many post hoc analyses performed on the large databases of the most important recent observational studies (ECLIPSE, CCLS, COPDgene), the demonstration that these different groups of GOLD 2011 classification are different for physiological and biological features is controversial.

We performed a detailed examination of a group of 83 patients with COPD of different severity who attended to a pulmonary rehabilitation programme. COPD was defined according to GOLD guidelines [1], and patients were referred for a pulmonary rehabilitation programme by pulmonary specialists in our area.

As a part of the baseline evaluation of the pulmonary rehabilitation programme, all patients underwent clinical (symptoms, exacerbation rate, comorbidities), nutritional (body mass index (BMI), fat-free mass index (FFMI) and functional (pre- and post-bronchodilator static and dynamic lung volumes, diffusing capacity, resting blood gas measurement, 6-min walking distance) assessment, according to the standard procedures. Furthermore, each patient collected a sputum sample induced by hypertonic/isotonic saline inhalation, which was processed for sputum cell analysis [6], and also an exhaled breath condensate sample for the measurement of malondialdehyde as a marker of oxidative stress [7]. Informed consent for participation to the study was obtained from all patients. The local ethics committee was advised of this observational study. All patients were examined in a stable phase of the disease and had not had an acute exacerbation for at least 4 weeks. Regular treatment was withdrawn $24 \mathrm{~h}$ before each examination.

Comparisons among groups were performed with ANOVA, with Student-Newman-Keuls multiple comparison test, for normally distributed variables, and with Kruskall-Wallis one-way analysis of variance, followed by post hoc Bonferroni Dunn's test, for non-normally distributed variables.

Patients were stratified in the four GOLD 2011 categories according to the three parameters: level of dyspnoea on exercise (mMRC scale), FEV1 \% predicted, and number of exacerbations in the last year (requiring oral corticosteroids and/or antibiotics) (table 1). The mean \pm sD age of the enrolled patients was $68.8 \pm 7.8$ years, 65 were males, 75 were smokers or ex-smokers; post-bronchodilator FEV1 \% pred was $54.1 \pm 19.0$ and $\mathrm{FEV} 1 /$ forced vital capacity was $48.0 \pm 12.3 \%$ of predicted values. There was no significant difference among the four groups for demographic data, except for BMI and FFMI which were higher in group A. Diffusing capacity was significantly lower, and total lung capacity higher, in B and D compared with $\mathrm{A}$ and $\mathrm{C}$. These data were associated with a poorer quality of life, as assessed by St George's Respiratory Questionnaire total score, in B and D. Group D showed the lowest mean value of arterial oxygen tension. Patients were under regular treatment with long-acting bronchodilators (long-acting $\beta_{2}$-agonist and/or long-acting muscarinic antagonist, $n=77$ ), inhaled corticosteroids $(n=66)$ and oral theophylline $(\mathrm{n}=15)$; no patient was under regular oral corticosteroid regimen. Groups B and D showed higher sputum neutrophil percentages than $\mathrm{A}$ and $\mathrm{C}$ groups, while sputum eosinophil percentages were significantly higher in A and C in comparison with B and D groups. Neutrophilia (neutrophils $>64 \%$ ) was present in $62(75 \%)$ patients and eosinophilia (eosinophils $>2 \%$ ) was present in $45(54 \%)$ patients $[8,9]$. No relationship was observed between sputum cell counts and pharmacological treatment. No significant difference among the four groups was observed for malondialdehyde levels in exhaled breath condensate. 
TABLE 1 Main characteristics of patients divided according to the GOLD 2011 classification

GOLD 2011 categories

\section{Patients $\mathrm{N}=83$}

GOLD 2011 assessment variables

mMRC dyspnoea score

FEV1 \% predicted

Exacerbations last year $n$

Demographics

Age years

Male

Smoking status

Ex-smoker

Current

Pack-years

Body mass index $\mathrm{kg} \cdot \mathrm{m}^{-2}$

Fat-free mass index $\mathrm{kg} \cdot \mathrm{m}^{-2}$

Charlson index

Physiology

TLC \%

DLCO \%

$D\left\llcorner\mathrm{LO} / V_{\mathrm{A}} \%\right.$

$\mathrm{PO}_{2}$

$\mathrm{PCO}_{2}$

SGRQ total \%

Pharmacological treatment

ICS therapy

LAMA/LABA therapy

Biomarkers

IS neutrophils $10^{6}$ cells $\mathrm{mL}^{-1}$

IS neutrophils \%

IS neutrophilia

IS eosinophils $10^{6}$ cells $\cdot \mathrm{mL}^{-1}$

IS eosinophils \%

IS eosinophilia

EBC MDA nM

A

\begin{tabular}{|c|c|c|c|}
\hline $15(18.1)$ & 10 (12.0) & $20(24.1)$ & 38 (45.8) \\
\hline $0.7 \pm 0.5$ & $2.5 \pm 0.7$ & $0.8 \pm 0.4$ & $2.9 \pm 0.7$ \\
\hline $66.8 \pm 11.7$ & $69.0 \pm 15.6$ & $54.7 \pm 16.3$ & $44.8 \pm 18.6$ \\
\hline $0.5 \pm 0.5$ & $0.4 \pm 0.5$ & $2.4 \pm 1.9$ & $2.8 \pm 1.8$ \\
\hline $69.5 \pm 6.8$ & $73.8 \pm 6.8$ & $68.6 \pm 7.4$ & $69.5 \pm 8.5$ \\
\hline 13 (87) & $5(50)_{\mathrm{s}}$ & $18(90)$ & $29(76)$ \\
\hline $12(80)$ & $8(80)$ & $14(70)$ & $25(66)$ \\
\hline $2(13)$ & $0(0)$ & $4(20)$ & $10(26)$ \\
\hline $48.3 \pm 26.7$ & $60.2 \pm 28.8$ & $39.3 \pm 22.9$ & $51.2 \pm 23.3$ \\
\hline $29.7 \pm 5.1$ & $26.7 \pm 4.8$ & $26.0 \pm 4.7$ & $27.1 \pm 4.8$ \\
\hline $20.8 \pm 2.7$ & $17.9 \pm 5.4$ & $16.9 \pm 2.2$ & $17.8 \pm 2.8$ \\
\hline $1.7 \pm 1.6$ & $2.2 \pm 0.7$ & $1.6 \pm 1.3$ & $1.5 \pm 1.2$ \\
\hline $105.4 \pm 9.4$ & $123.7 \pm 15.8$ & $119.7 \pm 25.2$ & $132.3 \pm 29.2$ \\
\hline $86.3 \pm 16.3$ & $58.4 \pm 19.4$ & $91.3 \pm 27.6$ & $58.6 \pm 24.5$ \\
\hline $113.5 \pm 20.0$ & $80.1 \pm 32.2$ & $110.9 \pm 24.7$ & $87.4 \pm 27.7$ \\
\hline $78.3 \pm 15.0$ & $77.1 \pm 7.7$ & $74.6 \pm 9.9$ & $69.7 \pm 9.0$ \\
\hline $38.4 \pm 4.8$ & $36.4 \pm 5.6$ & $40.9 \pm 4.7$ & $42.1 \pm 6.5$ \\
\hline $25.4 \pm 9.7$ & $43.7 \pm 14.7$ & $35.7 \pm 11.0$ & $58.7 \pm 16.6$ \\
\hline $6(40)$ & 7 (70) & $17(85)$ & 36 (95) \\
\hline $11(73)$ & $9(90)$ & 19 (95) & $38(100)$ \\
\hline $2.2(0.1-7)$ & $4.9(1-60)$ & $2.7(0.1-15)$ & $2.4(0.1-14)$ \\
\hline $66.5(15-91)$ & 84.2 (58-99) & $72.1(18-88)$ & 78.7 (44-97) \\
\hline $7(46)$ & $9(90)$ & $12(60)$ & 34 (89) \\
\hline $0.06(0-1.1)$ & $0.07(0-0.4)$ & $0.3(0-5)$ & $0.03(0-0.7)$ \\
\hline $2.6(0.4-21)$ & $1.4(0-9)$ & $2.9(0-60)$ & $1.1(0-23)$ \\
\hline $11(73)$ & $4(40)$ & $15(75)$ & 15 (39) \\
\hline $17.2(4-68)$ & $17.1(4-33)$ & $25.5(4-81)$ & $26.3(6-126)$ \\
\hline
\end{tabular}

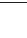

B

C

D

D

Anova/Chi-squared/Kruskall Wallis + post hoc analysis $p<0.0001 ; A, C$ versus $B, D$
$p<0.0001 ; A, B$ versus $C, D$
$p<0.0001 ; A, B$ versus $C, D$

NS; $p=0.08$

NS; $p=0.33$

NS; $p=0.27$

$\mathrm{p}<0.05$; B versus C

$\mathrm{p}<0.05$; A versus $C$

$\mathrm{p}<0.01$; $A$ versus $C, D$ NS; $p=0.08$

$\mathrm{p}<0.01$; $\mathrm{A}$ versus $\mathrm{B}, \mathrm{D}$

$p<0.01 ; A, C$ versus $B, D$

$\mathrm{p}<0.05 ; A, C$ versus $B, D$

$p<0.05$; $D$ versus $A, B, C$

$\mathrm{p}<0.05$; $B$ versus $D$

$p<0.01 ; A$ versus $B, C, D C$ versus $D$

$p<0.001$

NS; $p=0.25$

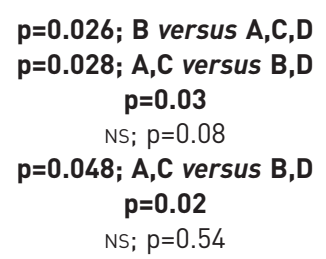

Data are reported as $\mathrm{n}(\%)$, mean \pm SD or median (range), unless otherwise stated. GOLD: Global Initiative for Chronic Obstructive Lung Disease; mMRC: modified Medical Research Council; FEV1: forced expiratory volume in $1 \mathrm{~s}$; TLC: total lung capacity; DLCo: single-breath diffusing capacity of the lung for carbon monoxide; $V$ A: alveolar volume; $P_{2}$ : oxygen tension; $P_{\mathrm{CO}_{2}}$ : carbon dioxide tension; SGRQ: St George's Respiratory Questionaire; ICS: inhaled corticosteroids; LAMA/LABA: bronchodilator long-acting muscarinic antagonist/long-acting $\beta 2$-agonist; IS: induced sputum; EBC: exhaled breath condensate; MDA: malondialdehyde. Ns: nonsignificant. A value of $p<0.05$ was considered as significant.

Our observation, although performed on a small group of COPD patients attending to an outpatient Pulmonary Rehabilitation Programme, confirms that other physiologic and biologic differences, apart from those used for the new GOLD classification, can be observed among the A, B, C and D groups. As expected, markers of emphysema (like tendency to have pulmonary hyperinflation, and reduction in diffusing capacity) were higher in $\mathrm{B}$ and $\mathrm{D}$ groups with higher dyspnoea score, confirming that dyspnoea in COPD patients is mainly related to the level of mechanical abnormalities due to the emphysema component of COPD [10]. In the much larger cohort of the ECLIPSE study, emphysema as assessed by computed tomography evaluation, was more evident in $\mathrm{C}$ and $\mathrm{D}$ categories, while there was no difference between A and B categories. The higher dyspnoea score in group B in comparison with group A of the ECLIPSE study was explained by the higher number of comorbidities in group B (mainly cardiovascular comorbidities) which may increase the level of dyspnoea for other non-pulmonary limitations. Our study examined a limited number of patients, and furthermore they had been selected from the possibility to attend to an outpatient Pulmonary Rehabilitation Programme; therefore severe cardiovascular diseases, which might represent an exclusion criteria for the exercise training, were excluded.

In our experience, Group B and D showed the higher neutrophil percentage in the induced sputum, while eosinophil percentages were higher in $\mathrm{A}$ and $\mathrm{C}$ groups in comparison with $\mathrm{B}$ and $\mathrm{D}$ groups. This observation might suggest that sputum neutrophilia is really associated with a higher level of COPD 
severity. While sputum neutrophilia is a characteristic feature of all COPD patients, independently from the specific clinical phenotype, sputum eosinophilia seems to be more frequent in a "bronchitic phenotype" with lower level of symptoms and markers of emphysema. At our knowledge, this is new information, not reported in the GOLD 2011 classification. Sputum and blood neutrophilia has been reported as a marker of severity of COPD, according to FEV1 or quality of life measurement [11], while sputum eosinophilia has been demonstrated in subgroups of COPD patients [12] and it has been used as a biomarker potentially useful in the therapeutic decision (e.g. use or not of inhaled corticosteroids) [13].

In summary, our observation, although performed on a small group of selected COPD patients, suggests that the A, B, C and D groups of the new GOLD classification are at least partly related to a different COPD phenotype (prevailing emphysema in B and D groups with greater neutrophilic inflammation, prevailing chronic bronchitis $\mathrm{A}$ and $\mathrm{C}$ groups with greater eosinophilic inflammation). This information may contribute to a better characterisation and possibly management of the wide range of categories included now in the COPD definition.

The A, B, C and D groups of the new GOLD classification are at least partly related to different COPD phenotypes http://ow.ly/Xgr8q

Maria Laura Bartoli, Francesco Costa, Laura Malagrinò, Dario Nieri, Sandra Antonelli, Giovanna Decusatis, Claudia De Simone, Sabrina Santerini, Silvana Cianchetti, Manuela Latorre, Barbara Vagaggini and Pierluigi Paggiaro

Cardio-Thoracic and Vascular Department, Respiratory Pathophysiology and Rehabilitation Unit, University Hospital of Pisa, Italy.

Correspondence: Maria Laura Bartoli, Cardio-Thoracic and Vascular Department, University of Pisa, Ospedale di Cisanello, Via Paradisa 2, 56124 Pisa, Italy. E-mail: m.bartoli@ao-pisa.toscana.it

Received: May 192015 | Accepted after revision: Oct 292015 | First published online: Feb 042016

Conflict of interest: Disclosures can be found alongside the online version of this article at erj.ersjournals.com

\section{References}

1 Global Initiative for Chronic Obstructive Lung Disease. Global strategy for the diagnosis, management, and prevention of chronic obstructive pulmonary disease. Available from www.goldcopd.org/uploads/users/files/ GOLD_Report_2015_Apr2.pdf Date last updated: 2015. Date last accessed: Nov 112015.

2 Han MK, Muellerova H, Curran-Everett D, et al. GOLD 2011 disease severity classification in the COPDGene study: a prospective cohort study. Lancet Respir Med 2013; 1: 43-50.

3 Lange P, Marott JL, Vestbo J, et al. Prediction of the clinical course of chronic obstructive pulmonary disease, using the new GOLD classification. Am J Respir Crit Care Med 2012; 186: 975-981.

4 Soriano JB, Alfageme I, Almagro P, et al. Distribution and prognostic validity of the new GOLD grading classification. Chest 2013; 143: 694-702.

5 Agusti A, Edwards LD, Celli B, et al. Characteristics, stability and outcomes of the GOLD 2011 COPD groups in the ECLIPSE cohort. Eur Respir J 2013; 42: 637-647.

6 Cianchetti S, Bacci E, Bartoli ML, et al. Can hypertonic saline inhalation influence preformed chemokine and mediator release in induced sputum of chronic obstructive pulmonary disease patients? Comparison with isotonic saline. Clin Exp Allergy 2007; 37: 1819-1826.

7 Bartoli ML, Novelli F, Costa F, et al. Malondialdehyde in exhaled breath condensate as a marker of oxidative stress in different pulmonary diseases. Mediators Inflamm 2011; 2011: 891752.

8 Belda J, Leigh R, Parameswaran K, et al. Induced sputum cell counts in healthy adults. Am J Respir Crit Care Med 2000; 161: 475-478.

9 Davidson WJ, The S, Leigh R. Establishing a normal range for induced sputum cell counts in Western Canada. Can Respir J 2013; 20: 424-425.

10 Langer D, Ciavaglia CE, Neder JA, et al. Lung hyperinflation in chronic obstructive pulmonary disease: mechanisms, clinical implications and treatment. Expert Rev Respir Med 2014; 8: 731-749.

11 Singh D, Edwards L, Tal-Singer R, et al. Sputum neutrophils as a biomarker in COPD: findings from the ECLIPSE study. Respir Res 2010; 11: 77.

12 Saha S, Brightling CE. Eosinophilic airway inflammation in COPD. Int J Chron Obstruct Pulmon Dis 2006; 1: $39-47$.

13 Brightling CE, Monteiro W, Ward R, et al. Sputum eosinophilia and short-term response to prednisolone in chronic obstructive pulmonary disease: a randomised controlled trial. Lancet 2000; 356: 1480-1485. 